\title{
Pregnancy and Covid-19 in Mexico, risk and associated comorbidities: A case-control study.
}

\author{
Eduardo Hernández-Garduño ${ }^{1}$ \\ ${ }^{1}$ Instituto de Seguridad Social del Estado de Mexico y Municipios
}

June 23, 2020

Title Page

Title: Pregnancy and Covid-19 in Mexico, risk and associated comorbidities: A case-control study.

Author: Eduardo Hernández-Garduño

Affiliation: Dirección de Administración y Desarrollo de Personal

Institution: Instituto de Seguridad Social del Estado de México y Municipios (ISSEMyM).

Corresponding author:

Eduardo Hernández-Garduño MD, MHSc

Departamento de Seguridad e Higiene ISSEMyM

Av. Constituyentes \#703

Col. Barrio de la Merced

Toluca de Lerdo, Estado de México

CP 50080

México

email: epidemiologist.researcher@gmail.com

Running title: comorbidities in pregnancy and risk for Covid-19

Word count: 1,699

\section{Hosted file}

Covid-19 and pregnancy in Mexican main document.docx available at https://authorea.com/ users/334488/articles/461738-pregnancy-and-covid-19-in-mexico-risk-and-associatedcomorbidities-a-case-control-study

\section{Hosted file}

Table 1.xlsx available at https://authorea.com/users/334488/articles/461738-pregnancy-andcovid-19-in-mexico-risk-and-associated-comorbidities-a-case-control-study

\section{Hosted file}

Table 2.xlsx available at https://authorea.com/users/334488/articles/461738-pregnancy-andcovid-19-in-mexico-risk-and-associated-comorbidities-a-case-control-study 